\title{
Structural Evidence of a Sealed Tissue Interface with a Total-etch Wet-bonding Technique in vivo
}

\author{
F.R. Tay, A.J. Gwinnett ${ }^{2}$, K.M. Pang ${ }^{1}$, and S.H.Y. Wei \\ Department of Children's Dentistry and Orthodontics, and 'Oral Biology Unit, Prince Philip Dental Hospital, The University of Hong Kong, \\ 34 Hospital Road, Hong Kong; and 2 Department of Oral Biology and Pathology, School of Dental Medicine, State University of New York \\ at Stony Brook, New York 11794-8702
}

\begin{abstract}
The resin-dentin interface of in vivo specimens restored with the All-Bond 2 system by use of a total-etch wetbonding technique on vital deep human dentin was investigated by: (a) SEM examination of cryof ractured specimens; (b) SEM examination of acid-rinsed, highly polished, embedded specimens; and (c) TEM examination of demineralized ultrathin sections. Ultrastructuralfeaturesthat were pertinent to the formation of an effective clinical seal were characterized. It is suggested that the establishment of an effective seal of the patent dentinal tubules following total etching is accomplished by: (1) the formation of an outer zone of a solid resin plug surrounded by a circumferential cuff of resin-impregnated dentin; and (2) the formation of an inner zone of a hollow resin sheath with resin globules along the internal walls of the tubules, closely adapting to the odontoblast process. The structural appearance andf unctional implications of these two zones were strikingly similar, with the sealing of exposed dentinal tubules in arrested carious lesions or cervical abfraction lesions. It appeared that there is a common denominator in physiologic isolation defense mechanisms and the total-etch, wet-bonding technique in the All-Bond 2 system in preserving the biological and morphological integrity of the pulpo-dentinal complex.
\end{abstract}

Key words. Total Etch, Dentin Bonding, Seal.

\section{Introduction}

Research directed toward achieving durable adhesion between polymeric restorative materials and dentinal surfaces has been progressing rapidly within the past decade. While clinical success has been achieved with respect toenamel surfaces, the development of dentin bonding agents has been a challenge and is presently in its fourth generation of evolution. The development and commercialization of thefirst generation of dentinadhesives occurred in the early 1960's (Bowen, 1965; Crim et al., 1984; Beech, 1985). Apart from the quest for optimal assembly bond strength to the underlying substrate, a central goal has been the objective of securing intimate adaptation of the restorative material with the cavity interfaces to resist microleakage and the influx of oral pathogens or their products, which may lead to post-operative sensitivity, interfacial staining, and recurrent caries.

The first and second generations of dentin adhesives were based upon interactions with the smear layer. Historically, such a layer was believed to provide a protective role as an iatrogenically produced cavity liner that protected the pulp from the effect of microleakage. However, viable micro-organisms have been shown to be retained and tocolonize within the smear layer (Brännström and Nyborg, 1973). Their byproductsmaydiff use through the fluid-filled channelspresent within the solid phase of cutting debris (Pashley et al., 1992). Bacterial ingrowth, for example, could explain why a number of the Class V lesions restored with Scotchbond (3M, St. Paul, MN) resulted in pulpal necrosis (Vanherle et al., 1986).

A total-etch technique, in combination with improved formulations of hydrophilic primers and resins, has been advocated as a safe and effective method to achieve significant adhesion to dentin (Bertolotti, 1991; Kanca, 1991; Fusayama, 1992). Such a technique has recently been adopted as a standard protocol for the latest generation of dentin adhesive systems, such as All-Bond 2 (Bisco, Itasca, IL) and Scotchbond 
Multipurpose (3M, St. Paul, MN). Moreover, it has been reported that a significant reduction in microleakage, without compromise in bond strength, has been observed in the Syntac system (Vivadent, Schaan, Liechtenstein), when a total-etch technique was compared with the manufacturer's recommendation (Gwinnett et al., 1992).

It is pragmatic to compare freshly cut, acid-conditioned dentin, with an open wound, with the channels of communication to the connective tissue pulp via the dentinal tubules. While the total-etch technique removes the smear layer to optimize adhesion through hybridization, the debris which normally occludes the entrance to the dentinal tubules (smear plugs) is also removed. Unless these patent tubules are effectively sealed, a dynamic exhange of dentinal fluids, bacteria, and their products can occur along the tooth-restoration interface. This uncontrolled flow of fluid through the dentinal tubule compartment-which may leave the subject sensitive to thermal, osmotic, and atmospheric stimulus-is manifested as the sensory component of microleakage (Pashley, 1985; Cox, 1992a). It can be explained by the hydrodynamic theory of dentin sensitivity (Brännström et al.,1967). The resultant effect of microleakage is the diffusion of bacterial products through the dentinal tubule complex, which of ten results in pulpal inflammation and eventual periapical pathology. There is ample evidence to show that bacteria at the restoration/tissue interface are the principal cause of pulpal irritation from dental restorative materials (Crowell, 1927; Kakehashi et al., 1965; Bergenholtz, 1977, 1990; Brännström et al., 1979; Qvist, 1980; Brännström, 1985; Cox et al., 1987).

The open wound analogy is also supported by observations that open dentinal tubules exist in abundance in exposed hypersensitive radicular dentin (Yoshiyama et al.,1989,1990). This allowsfluid movement across the tubules, thus stimulating nerves either in the pulpal half of the dentin and/or in the pulp itself, to elicit a painf ul response. Acid demineralization and enlargement of dentinal tubules were observed in active caries lesions (Arends et al., 1989), creating a pathway for the invasion of pioneer micro-organisms (MacGregor et al., 1956; Ohgushi and Fusayama, 1975). Does the current generation of dentin adhesives contribute an effective seal against pathological consequences so as to preserve the biological integrity of the pulpal-dentinal complex? The purpose of thisstudy is to present the initial structural evidence for a total seal in vivo with the use of the All-Bond 2 adhesive system.

\section{Materials and methods}

Twenty previously unrestored bicuspids, being removed for orthodontic reasons, were obtained from five young adults with informed consent. Prior to the commencement of the clinical protocol, these teeth were examined clinically and radiographically to ensure absence of caries or cervical attrition. Deep, unexposed cylindrical Class $V$ cavities of about 4 $\mathrm{mm}$ in diameter were prepared on the buccal surface of each bicuspid by means of ultra-high speed and copious water cooling. Local anesthesia (with vasoconstrictor) and rubber dam application were used. The occlusal margin was placed on enamel, and the gingival margin was as close to the buccal gingiva as possible without trauma. A $90^{\circ}$ cavosurface margin was maintained at both the enamel and gingival sites. Cavity depth was judged by clinical observation of a thin translucent layer of remaining dentin on the cavity floor overlying the pulp as well as by measurement with a periodontal probe.

The prepared cavities were etched with $10 \%$ phosphoric acid (All-Etch Semi-Gel, Bisco, Itasca, II)for 20 sand briefly dried with a triple syringe, leaving the surfacesslightly moist. They were then restored with the All-Bond 2 universal adhesive system (Bisco, Itasca, IL)according tothemanufacturer'sinstructions. Onedropof primer A(NTG-GMA)and primer B(BPDM)wasdispensed, mixed in a plastic well, and applied to the conditioned cavity in 5 consecutive coats. There was nodrying of the mixed primer between coats. The primer was then dried under a gentle flow of air, and it was carefully noted that a glossy film remained on the surface of the dentin. A thin film of enamel and dentin bonding resin from the All-Bond 2 kit was applied with a brush to the primed surface and polymerized for $20 \mathrm{~s}$. The cavity was restored with Z100 (3M, St Paul, MN) by an incremental placement technique. All teeth were left tof unction between 21 and 28 days beforeextraction. Extracted teeth were immediately severed at the middle of the root to expose the root canals in order to facilitate the penetration of the fixative. They were immersion-fixed with $10 \%$ neutral buffered formalsaline (NBFS) for $48 \mathrm{~h}$. After fixation, all teeth were rinsed thoroughly in running water for $8 \mathrm{~h}$ or overnight.

\section{Cryof racture preparation for SEM examination}

In half of the fixed specimens, a shallow slit of about $2 \mathrm{~mm}$ was made longitudinally over the center of the cavity preparation with a rotating diamond-impregnated copper disc. The teeth were then cryof ractured by immersion in liquid nitrogen for $10 \mathrm{~min}$ and pressure applied with a scalpel through the preformed slit. The fractured portions were rinsed repeatedly in distilled water and then post-fixed in $1 \%$ osmium tetroxide (TAABLaboratories, England)for $2 \mathrm{~h}$ at $4^{\circ} \mathrm{C}$. They were subsequently dehydrated by being passed through a graded ethanol series, dried in a critical-point dryer (CPD-020, Balzer,Switzerland) with liquid carbon dioxideasa transitional medium. The dried fragments were mounted onto metal stubs with colloidal carbon adhesive and coated wih gold in an ion sputtercoater (FC-1100, JEOL, Japan) at $1.2 \mathrm{kV}$ and $5 \mathrm{~mA}$ for $8 \mathrm{~min}$. Observations were performed in a scanning electron microscope (JXA-840, JEOL, Japan) operating at 10-15 kV.

\section{Preparation for SEM/TEM observations}

The remaining specimens were sectioned buccolingually through the mid-portion of the cavity preparation by means of a low-speed cutting machine equipped with a diamond-impregnated copper disc under copious water irrigation. The hemi-sectioned teeth were post-fixed in $1 \%$ osmium tetroxidefor $2 \mathrm{~h}$ at $4^{\circ} \mathrm{C}$, dehydrated in a graded ethanol series, cleared in propylene oxide, infiltrated in epoxy resin (TAAB 812 resin, TAAB Laboratories, 


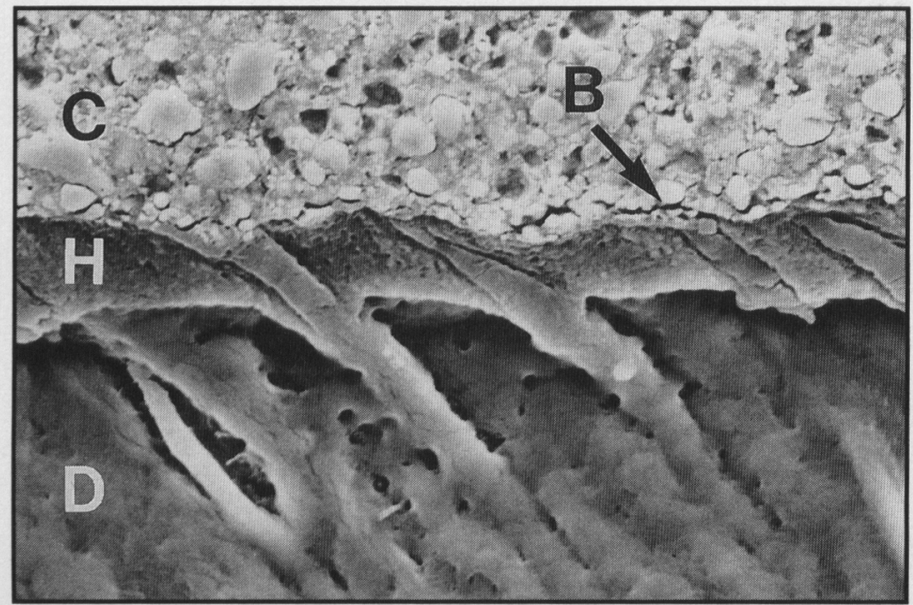

Figure 1. The hybrid layer as it appeared in highly polished, acidrinsed specimens. Initial constrictions of some, but not all, of the resin strings were observed at the entrance to the hybrid layer. C, composite $\mathrm{B}$ (arrowed), primer/bonding resin; $\mathrm{H}$, hybrid layer; $\mathrm{D}$, dentin. $\mathrm{x} 3000$

England), and finally embedded in pure epoxy resin. After polymerization for $24 \mathrm{~h}$ at $60^{\circ} \mathrm{C}$, resin blocks were ground with silicon carbide abrasive cloth (CC 1200 CW, OS Abrasive, OSA, Germany), with running water as lubricant, to expose the specimen surface and remove cut marks and initial scratches. The ground surfaces were highly polished on alumina-coated lapping film (3M,St. Paul, MN) and then acid-rinsed in $0.01 \mathrm{~mol} / \mathrm{L} \mathrm{HCl}$ for $20 \mathrm{~s}$ for removal of the smear layer, bringing the interface into relief through partial dissolution of the dentin. The etched specimens were washed thoroughly in distilled water and dried in a criticalpoint-dryer. They were secured onto metal stubs with colloidal carbon adhesiveand coated with gold in an ion-sputterer at $1.2 \mathrm{kV}$ and $5 \mathrm{~mA}$ for $8 \mathrm{~min}$. Observations were performed in a scanning electron microscope(JXA-840,JEOL,Japan)operating at 10-15kV.

During SEM examination, representative areas of interest along the resin-dentin interface were recorded with photos or sketches. These selected regions were then trimmed out from the specimens as small rectangular blocks of approximately 1 $\mathrm{mm}^{2}$ in cross-section, and further processed for block demineralization by immersion in Kristensen's fluid (1N sodium formate in $8 \mathrm{~N}$ formic acid, $\mathrm{pH}=2.2$ ) for $18 \mathrm{~h}$. The decalcified blocks were washed thoroughly in running water and then in distilled water, dehydrated in a graded ethanol series, and re-embedded in epoxy resin (TAAB 812 resin, TAAB Laboratories, England). Ultrathin sections of approximately $90-100 \mathrm{~nm}$, as judged by their interference colors, were prepared with an ultramicrotome (LKB Ultrotome, Germany). They were picked up on 300mesh formvar-coated copper grids (TAAB Laboratories, England). The grids were then double-stained with uranyl acetate lead citrate, and examined with a transmission electron microscope (JEM-100, JEOL, Japan) operating at $80 \mathrm{kV}$.

\section{Results}

The SEM photomicrographs of the highly polished and



Figure 2. Separation of the hybrid layer $(\mathrm{H})$ from the bonding resin above in an acid-rinsed specimen, permitting a better perspective of the fibrous surface of the hybrid layer. $\mathrm{x} 4000$.

cryof ractured specimens provided complementary information on the characterization of the resin-dentin interface, which was conf irmed and further resolved by the TEM observations.

A layer of resin-impregnated dentin (hybrid layer) was clearly revealed along the resin-dentin interface in the highly polished, acid-rinsed specimens under the SEM, measuring from 2 to 8 micrometers in width (Fig. 1). This layer, which was brought into relief following acid-rinsing, remained insoluble and possessed a unique texture when compared with the overlying primer/resin. Solid cores of resin were observed as they traversed this interface, with constrictions identified for some but not all of the resin cores (Fig. 1). In some specimens prepared for SEM, tiny areas of artefactual separation occurred between the bonding resin and the intact hybrid layer as a result of the dehydration during laboratory processing and examination under vacuum, providing a better view of the latter feature (Fig. 2). The hybrid layer in cryofractured specimens appeared to have a distinctly homogenous resinous texture (Fig. 3).

The application of $10 \%$ phosphoric acid to vital dentin resulted in: (1) the removal of the smear plugs that normally occluded the entrances to the dentinal tubules; (2) widening of the orifices by complete removal of the highly mineralized peritubular dentin; and (3) theformation of funnel-shaped resin plugs(Fig. 4). Theseresin plugs havea very rough surfacetexture, seen also in cryof ractured specimens, with lateral branches that occupied the communicating channels between adjacent tubules. Examination of highly polished, acid-rinsed specimens revealed that these resin plugs comprised an outer circumferential cuff of resin-impregnated dentin that was continuous with the hybrid layer above (Fig. 5) and an internal solid core of resin, offering a more complete seal than was previously provided simply by the porous smear plug. "Sealing" or "a seal" is defined as the absence of gaps, voids, or tissue discontinuity with the resin.

Farther from the demineralized surface, extensions of the solid plugs now appeared as hollow resin strings. These were 


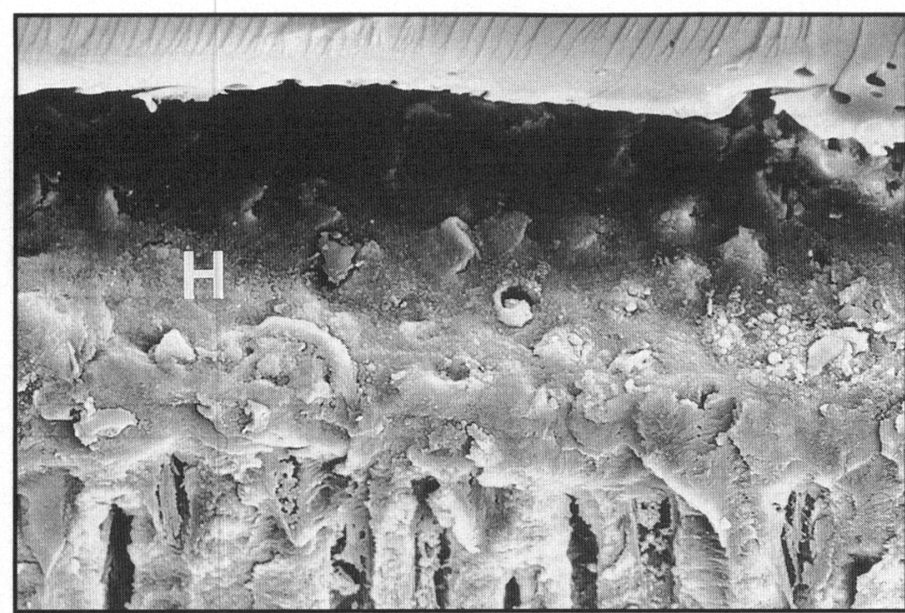

Figure 3. The hybrid layer $(\mathrm{H})$ as it appeared in a cryofractured specimen, revealing its pre-acid-rinsed, resinous texture. Sealing of the patent dentinal tubules by "fractured" resin cores within the hybrid layer is evident, despite the artef actual separation from the bonding resin above. $\mathrm{x} 2000$.

clearly revealed in cryof ractured specimens (Fig. 6), some of which could be seen to contain structures similar to odontoblast processes (Fig. 7). The internal surfaces of these hollow resin strings had granular deposits, and were seen clearly in cryof ractured specimens to consist probably of individual globular, resin droplets (Fig. 8) or aggregates (Fig. 9), the sizes of which decreased progressively away from the demineralized dentin surfaces (Fig. 10).

TEM examination of a typical resin string at the cavity floor in deep vital dentin (Fig. 11) showed the presence of two morphologically discrete zones. The outer zone consisted of a solid resin core surrounded by a circumferential cuff of resin-im-

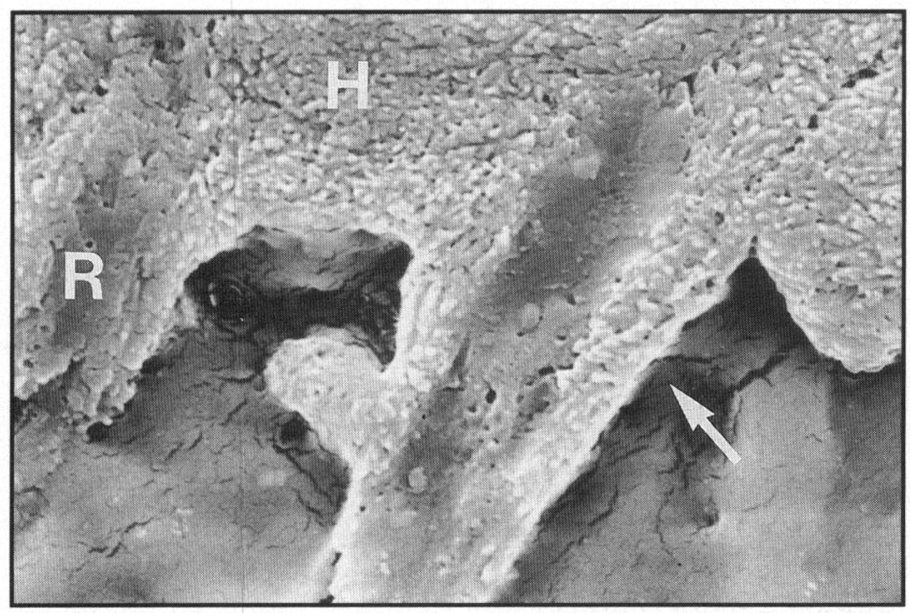

Figure 5. Longitudinal section through a resin plug from a highly polished acid-rinsed specimen, showing a solid core of resin (R) surrounded by an outer circumferential layer of resin-impregnated dentin (arrow) which is continuous with the surface hybrid layer $(\mathrm{H})$. $x 6500$

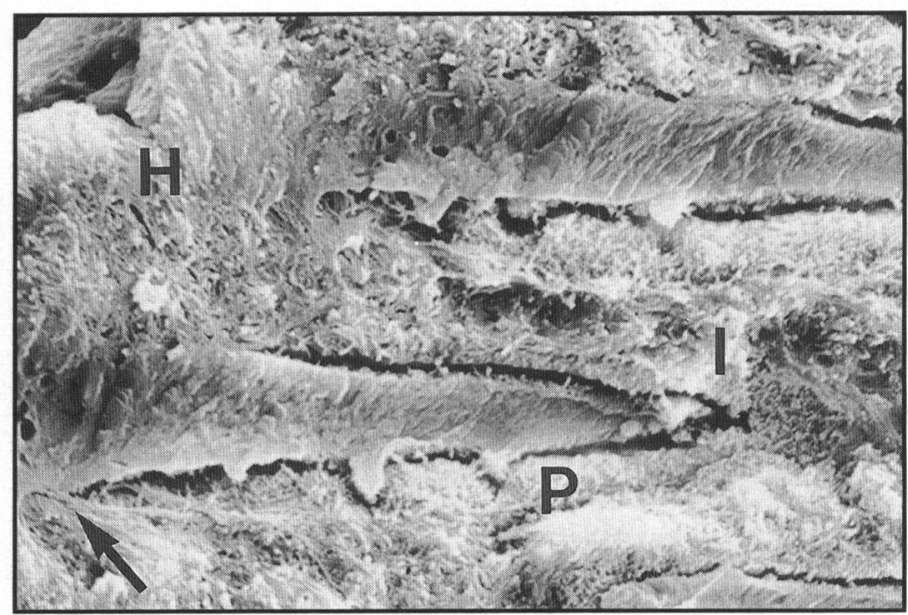

Figure 4. Funnel-shaped resin plugs from a cryof ractured specimen, demonstrating the rough surface texture and branches communicating between adjacent tubules. Conditioning with $10 \%$ phosphoric acid resulted in an initial complete dissolution of the peritubular dentin, with the formation of a layer of resin-impregnated intertubular dentin within the tubules that is continuous with the surf ace hybrid layer (arrow). Beyond this region, the amount of peritubular dentin gradually returned to normal. $\mathrm{H}$, hybrid layer; P, peritubular dentin; I, intertubular dentin. x10,000.

pregnated dentin, and appeared electron-dense. Within the solid resin core, a thinner, more electron-dense line was evident and was consistent with the lamina limitans observed in demineralized dentin. This demarcated the original dimension of the unconditioned dentinal tubule. The primer/resin material has occluded the space previously occupied by the smear plug (Fig.11), diff used through and incorporated the lamina limitans, and hasentered the space created by the complete dissolution of the highly inorganic peritubular dentin (Fig. 11). Resin also infiltrated the partially demineralized, intertubular dentin lat-

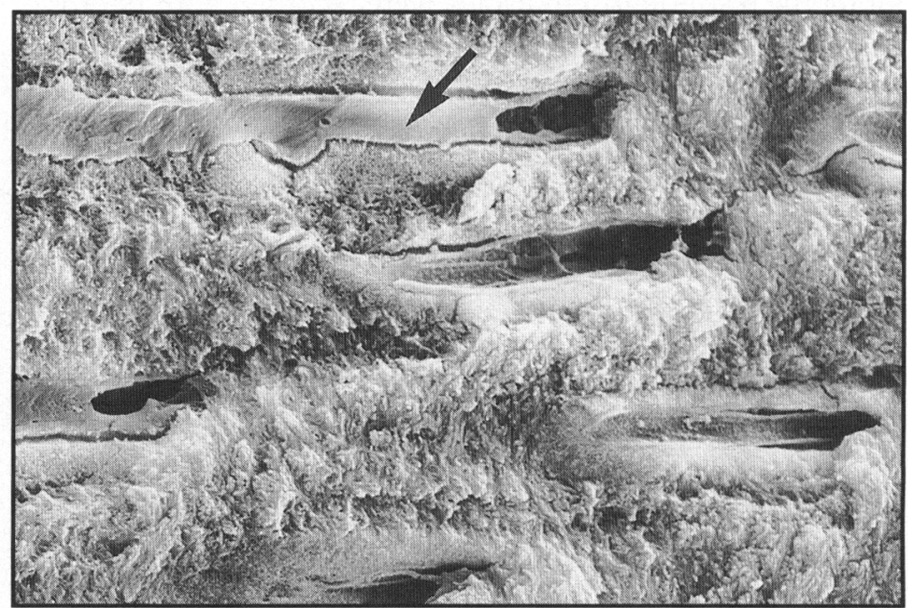

Figure 6. Hollow resin strings were invariably observed farther from the original demineralized dentin surface as it appeared in a cryofractured specimen. The full width of the adjacent peritubular dentin was apparently intact. The absence of a layer of resin-impregnated intertubular dentin resulted in a comparatively more placid external surface (arrow). x4000. 


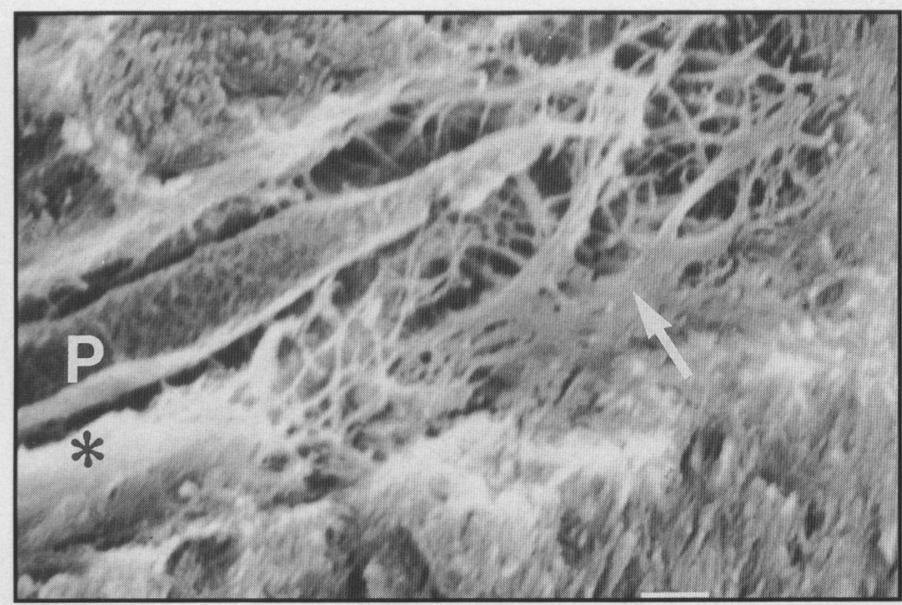

Figure 7.An obliquely fractured hollow resin string from a cryof ractured specimen, revealing a central structure that appeared to be an odontoblastic process $(\mathrm{P})$. Thisissurrounded by a hollow sheath of resin(asterisk) that appeared to have impregnated some of the loose, intratubular collagen present within the dentin tubule compartment (arrow). x8000.

erally with the formation of a circumferential layer of resinimpregnated intertubular dentin (Fig. 11). There was no separation present in the two layers of resin, the resin-impregnated intertubular dentin, or the immediately adjacent unaltered intertubulardentin. Thesespecimenswere subjected todesiccation during laboratory processing and the negative pressure created inside the vacuum chambers of the SEM and TEM. It is noteworthy that the primer/resin was stained with the uranyl acetate-lead citrate, while the embedding resin did not stain.

The outer zone was found to be continuous with the inner zone, where no resin-impregnated dentin could be further identified. Within the inner zone, a central odontoblast process surrounded by a hollow sheath of resin was of ten identified. An artefactual gap was evident between the resin sheath and the

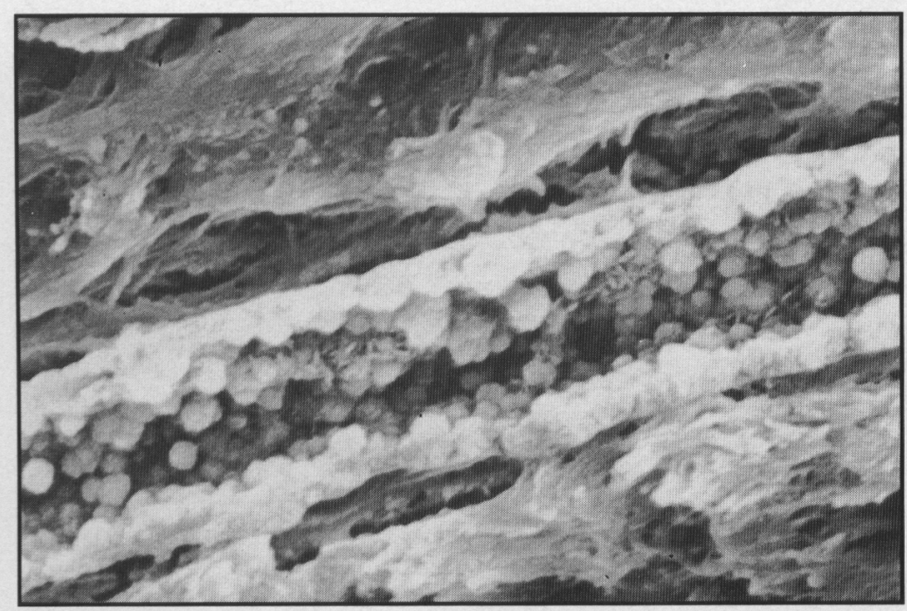

Figure 9.Longitudinal fracture through a thick, hollow resin string in a cryofractured specimen, showing a massive aggregation of resin globules that clustered predominantly along the internal surface. x12,000.

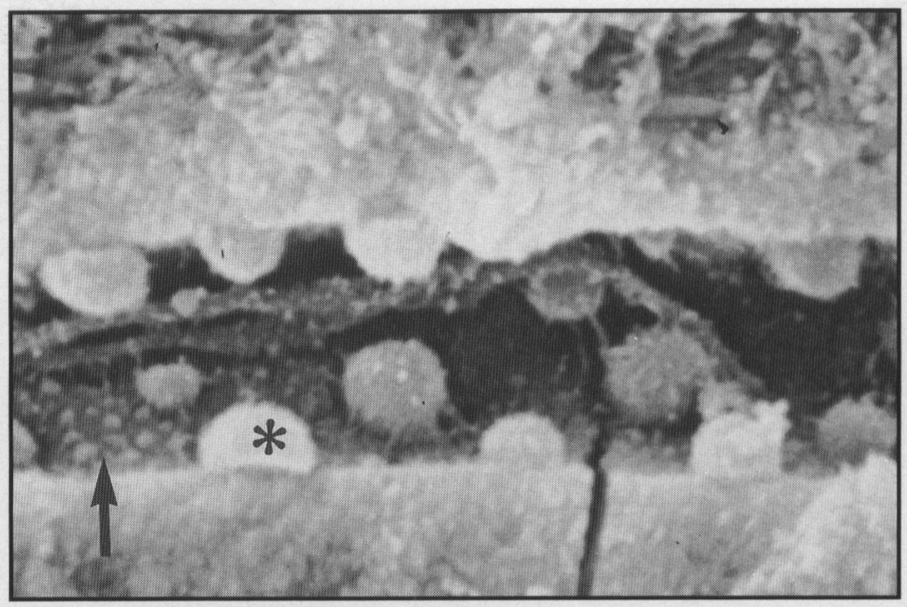

Figure 8. Large, individual, evenly spaced, spherical resin globules up to 0.7 micrometer in diameter (asterisk) were observed along the internal periphery of a hollow resin string. Interspersed among these globules were granules of much smaller size (arrow). Cryof ractured specimen. $\times 20,000$.

adjacent intertubular dentin, where the hybrid layer was absent. Numerous solid, semi-circular, resinous projections were found to fill the peri-odontoblastic space, adapting closely to the odontoblast process. The uniformity of staining reaction within the resinous material and the definite absence of organelles in these circular projections at an ultrastructural level excluded the possibility that the spherical granules observed in the SEM were micro-organisms. Further toward the periphery, the primer/ resin had infiltrated the region of the lamina limitans and filled the spaces created by the dissolution of the peritubular dentin.

\section{Discussion}

The removal of the smear layer has been considered essential

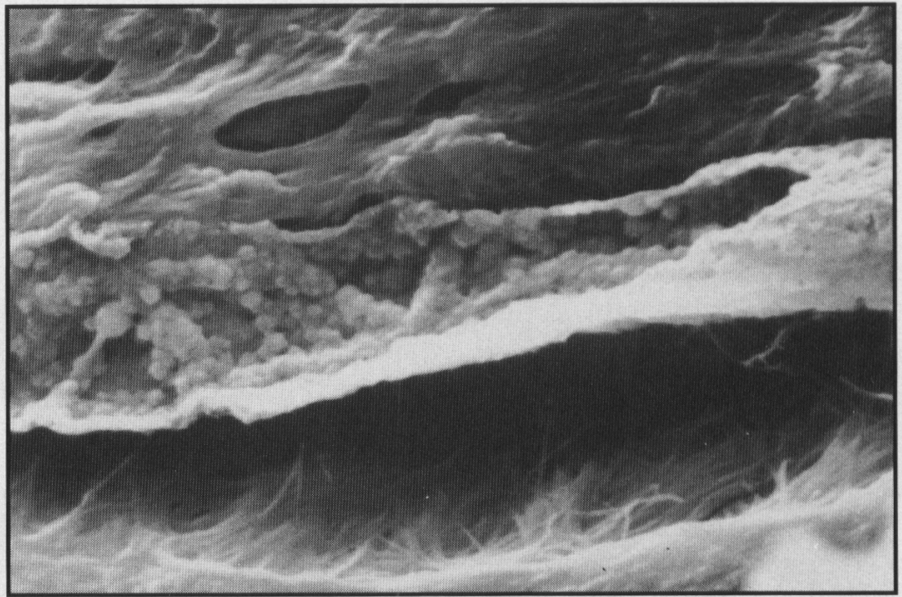

Figure 10. Compared with Fig. 9 from the same cryof ractured specimen, the thickness of the resin string was reduced, and the sizes of the resin globules became smaller farther from the demineralized dentinal surface. $\mathrm{x} 12,000$. 


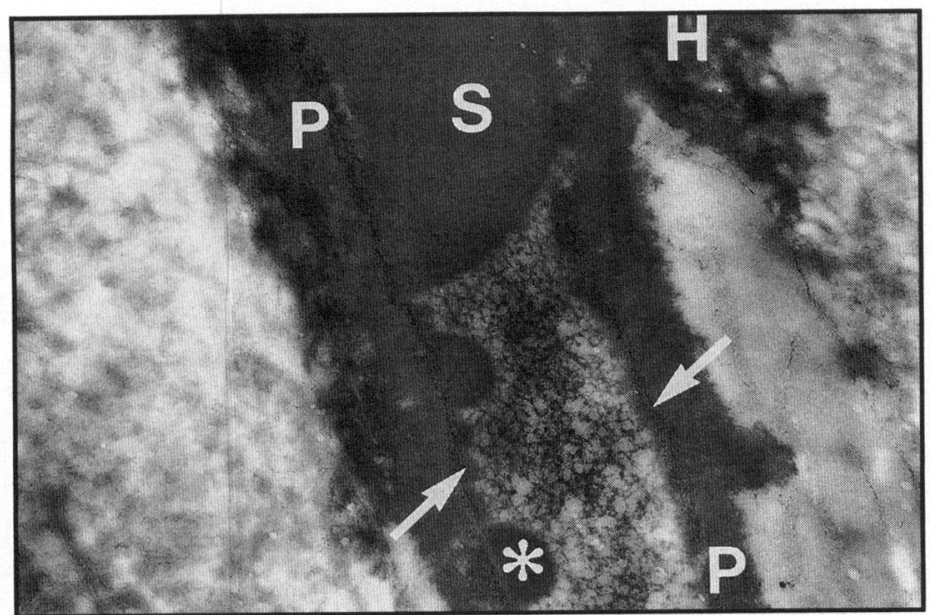

Figure 11. TEM view of a resin string at the junction between the solid resin plug (above) and the hollow resin string enveloping an odontoblast process (below). The initial solid seal following total etching consisted of three continuous layers: (i) resin replacing the original smear plug (S), (ii) resin occupying the spaces created by the dissolution of the peritubular dentin (P), and (iii) a circumferential layer of resin-impregnated intertubular dentin $(\mathrm{H})$. Within the hollow resin string further down, the resin globules (asterisk) corresponded with what was observed in Fig. 8 and were devoid of ultrastructural features that were suggestive of the presence of micro-organisms. The resin has infiltrated the lamina limitans (white arrow) and occupied the spaces created by the dissolution of the peritubular dentin (P). Little or no resin-impregnated intertubular dentin $(\mathrm{H})$ was observed along the periphery of the hollow resin string.(Note:Figs. 8 and 11 were photographed from separate specimens obtained from different subjects.) $\mathrm{Bar}=1 \mu \mathrm{m}$.

in optimizing the bond strength of dentin adhesive resins, and structural evidence has been provided to account for such a phenomenon (Gwinnett and Kanca, 1992a). The opening of the dentinal tubules by removal of the smear plugs, however, is more controversial (Rauschenberger, 1992), and there has been no convincing structural evidence, apart from indirect correlation from pulpal studies, to demonstrate the presence of a potentially biocompatible clinical seal. The present study describes and illustrates the main structural characteristics of the resin-dentin interface that are consistent with an effective clinical seal. In this case, a total-etch wet-bonding technique was used with the application of the All-Bond 2 system. No gaps or voids were observed. For the sake of description, the features were divided into: (1) an outer zone, consisting of a solid resin plug occluding the entrance to the dentinal tubules and a circumferential cuff of resin-impregnated intertubular dentin; and (2) an inner zone, consisting of a hollow resin sheath that completely filled the peri-odontoblastic space and the spaces created by the dissolution of the surrounding peritubular dentin. These features are represented schematically in Fig. 12.

The outer zone forms a barrier with the potential of blocking the entrance of micro-organisms to the underlying dentin. The existence of a hybrid layer has been demonstrated in human dentin in vivo (Nakabayashi et al.,199la; Gwinnett and Kanca, 1992b). Such a layer has been demonstrated in this

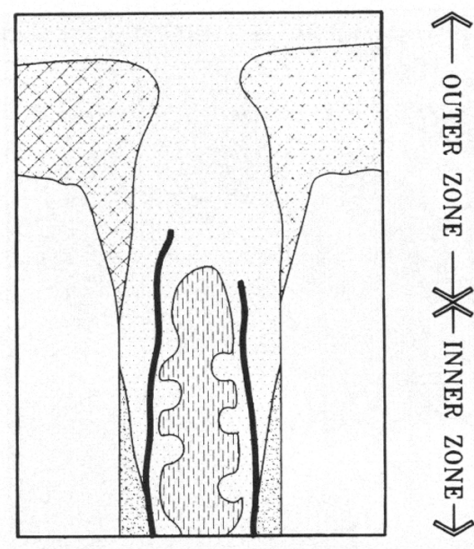

$\square$ PRIMER

HYBRII LAYER

$\square$ INTERTUBULAR DENTIN

PERITUBULAR DENTIN

EDONTOBLAST PROCESS

$\sim$ LAMINA LIMITANS

Figure 12. Schematic representation of the resin-dentin interface following a total-etch wet-bonding technique and the application of the All-Bond 2 system.

study as well as other studies (Inokoshi et al., 1990; Wang and Nakabayashi,1991; Van Meerbeek et al.,1993) to be insoluble in acid. The fact that the hybrid layer is acid-resistant affords a dual function of bonding a restoration to the tooth and rendering it caries-resistant (Nakabayashi et al.,1991b). In this regard, resin-dentin hybridization is analogous to etched and sealed enamel surfaces (Gwinnett and Matsui, 1967). Contrary to what was generally believed, such surfaces are more resistant to subsequent acid attack by the impregnation of insoluble resin tags into the enamel surfaces (Silverstone, 1982) as well as resin encapsulation of the individual crystallites, protecting the enamel from further dissolution(Gwinnett and Matsui, 1967). The incorporation of hydrophilic primers dissolved in acetone such as in the All-Bond 2 system dynamically permits resin infiltration into demineralized dentin as well as displacement of the dentinal fluid in vivo. An "outer acid-resistant, sealed zone" is thereby established in dentin to afford protection that was not conferred by the earlier generations of hydrophobic resins.

The filling of open tubules with polymerized resin alone does not necessarily constitute a seal. It has been speculated that polymerization shrinkage may result in loose-fitting resin tags, permitting fluid leakage in both directions under occlusal load (Pashley, 1991). It is unrealistic to expect that a dentin adhesive which relies solely on adhesion to demineralized collagen as its principal bonding mechanism will adhere to highly mineralized peritubular dentin. The formation of a circumferential layer of resin-impregnated intertubular dentin, however, between the resin core and the adjacent intertubular dentin probably affordsa bond at this critical junction.

The previously unreported features of the inner zone merits further discussion. It has been universally accepted that odontoblast processes were present within the inner one-third of vital dentin (Frank, 1966; Thomas, 1979; Maniatopoulos and Smith, 1983; Sigal et al., 1984; Weber and Zaki, 1986). The fact that the odontoblast process remained morphologically intact within the hollow resin sheath after 25 days offers prelimi- 
nary evidence on the biocompatibility of the dentin adhesive material being studied, further complementing the promising results reported in a recent pulpal study of the same system on primates (Cox, 1992b).

It was concluded that the acetone-based primer in the AllBond 2 system was able to wet the dentinal surface effectively. The acetone displaced the water and/or dentinalfluid in the in vivo situation, carrying with it the hydrophilic primers into the peri-odontoblastic space. Here, the resin formed a circular sheath of resin around the odontoblast process. This phenomenon, together with the intratubular collagen normally present in the dentinal tubules (Dai et al., 1991), could help to reduce the permeability of exposed dentin. According to the hydrodynamic theory, bulk fluid movement within patent dentinal tubules is responsible for the perception of external sensory stimuli, which is expressed as dentin pain. The replacement of the partially dissolved peritubular dentin and the normally fluid-filled peri-odontoblastic space with resin offers the possibility of reduced fluid transport across the resin-dentin interface. The clinical evidence presented in this study supports the potential use of the All-Bond 2 system as an effective seal and a dentin-desensitizing agent. The appearance of spherical droplets along the internal periphery of a resin string could possibly be explained by: (1) the polymerization of resinous material in a polar medium, taking up a surface configuration of the most energetically favored orientation (Suh, 1993, personal communication); and/or (2) initial micellar formation (Tanford,1980) of these primers, being surface-active comonomers with a hydrophilic head and a hydrophobic tail, acting subsequently as sites for further polymerization when more coats of primers are applied. Further research is indicated.

\section{Acknowledgments}

The technical assistance of Mr. Y.Y. Chui and Mr. Simon Lee of the Oral Biology Unit, The University of Hong Kong, is graciously acknowledged.

\section{References}

Arends J, Ruben J, Jongebloed WL (1989). Dentine caries in vivo. A combined SEM and microradiographic investigation. Caries Res 23:36-41.

Beech DR (1985). Bonding of restorative resins to dentin. In: Posterior composite resin dental restorative materials.Vanherle $\mathrm{G}$, Smith DC, editors.Utrecht: Peter Szulc Publishing Co., pp. 231-238.

Bergenholtz G (1977). Effect of bacterial products on inflammatory reactions in the dental pulp. Scand J Dent Res 85:122-129.

Bergenholtz G (1990). Pathogenic mechanisms in pulpal disease. J Endodont 16:98-101.

Bertolotti RL (1991). Total etch-the rational dentin bonding protocol. JEsthet Dent 3:1-6.

Bowen RL (1965). Adhesive bonding of various materials to hard tooth tissues: II. Bonding todentin promoted by a surface active comonomer. J Dent Res 44:895-902.
Brännström M(1985). Composite resin restorations: biological considerations with special reference to dentin and pulp. In: Posterior composite resin dental restorative materials. Vanherle G, Smith DC, editors. Utrecht: Peter Szulc Publishing Co., pp. 71-81.

Brännstrơm M, Linden LA, Åström A (1967). The hydrodynamics of the dental tubule and of pulpal fluid. A discussion of its significance in relation to dentinal sensitivity. Caries Res 1:310-317.

Brännström M,Nyborg H(1973).Cavity treatment with a microbicidal fluoride solution: growth of bacteria and effect on the pulp. $J$ Prosthet Dent 30:303-310.

Brännström M, Vojinovic O, Nordenwall KJ (1979). Bacterial and pulp reactions under silicate cement restorations.JProsthet Dent 41:290-295.

Cox CF (1992a). Microleakage related to restorative procedures. Proc Finn Dent Soc 88(Suppl 1):83-93.

Cox CF (1992b). Effects of adhesive resins and various dental cements on the pulp. Oper Dent (Suppl 5):165-176.

Cox CF, Keall CL, Keall HJ, Ostro E, Bergenholtz G (1987). Biocompatibility of surface-sealed dental materials against exposed pulps. JProsthet Dent 57:1-8.

Crim GA,SwartzML,PhillipsRW(1984). Anevaluation of cavosurface design and microleakage. Gen Dent 32:56-58.

Crowell J (1927). Physical chemistry of dental cements. J Am Dent Assoc 14:1013-1048.

Dai XF, Ten Cate AR, Limeback H (1991). The extent and distribution of intratubular collagen fibrils in human dentine. Arch Oral Biol 36:775-778.

Frank RM (1966). Etude au microscope electronique de l'odontoblaste et du canicule dentinaire humain. Arch Oral Biol 11:179-199.

Fusayama T (1992). Total etch technique and cavity isolation.J Esthet Dent 4:105-109.

Gwinnett AJ, Dickerson WG, Yu S (1992). Dentin bond shear strength and microleakage for Syntac/Heliomolar: A comparison between the manufacturer's and total etch technique.JEsthet Dent 4:164-168.

Gwinnett AJ, Kanca J III (1992a). Micromorphology of the bonded dentin interface and its relationship to bond strength. Am J Dent 5:73-77.

Gwinnett AJ, Kanca J III (1992b). Micromorphological relationship between resin and dentin in vitro and in vivo. Am J Dent 5:19-23.

Gwinnett AJ, Matsui A (1967). A study of enamel adhesives-the physical relationship between enamel and adhesive. Arch Oral Biol 12:1615-1620.

Inokoshi S, Hosoda H, Harniratissai C, Shimida Y, Tatsumi T (1990). A study on the resin impregnated layer of dentin: Part I. A comparative study on the decalcified and undecalcified sections and the application of argon ion beam etching to disclose the resin impregnated layer of dentin. Jpn J Conserv Dent 33:427-442.

Kakehashi S, Stanley HR, Fitzgerald RJ (1965). The effects of surgical exposures of dental pulps in germ-free and conventional laboratory rats. Oral Surg Oral Med Oral Pathol 20:340-349.

Kanca J III (1991). A method for bonding to tooth structure using phosphoric acid as a dentin-enamel conditioner. Quintessence Int 22:285-290.

MacGregor A, Marslan EA, Batty I (1956). Experimental studies of dental caries. I. The relation of bacterial invasion to sof tening of the dentine. Br Dent J 101:230-235. 
Maniatopoulos C, Smith DC (1983). A scanning electron microscopic study of the odontoblast process in human coronal dentine. Arch Oral Biol 28:701-710.

Nakabayashi N, Ashizawa M, Nakamura M (1991a). Identification of a resin-dentin hybrid layer in vital human dentin created in vivo: durable bonding to vital dentin. Quintessence Int 23:135-141.

Nakabayashi N, Nakamura M, Yasuda N (1991b). Hybrid layer as a dentin-bonding mechanism.J Esthet Dent 3:133-138.

Ohgushi K, Fusayama T (1975). Electron microscopic structure of the two layers of carious dentin. J Dent Res 54:1019-1026.

Pashley DH (1985). Dentin-predentin complex and its permeability: physiologic overview. J Dent Res 64(Spec Iss):613-620.

Pashley DH (1991). Clinical correlations of dentin structure and function.JProsthet Dent 66:777-781.

Pashley DH, Horner JA, Brewer PD (1992). Interactions of conditioners on the dentin surface. Oper Dent (Suppl 5):137-150.

Qvist V (1980). Correlation between marginal adaptation of composite resin restorations and bacterial growth in cavities. Scand J Dent Res 88:296-300.

Rauschenberger CR (1992). Dentin permeability. The clinical ramifications. Dent Clin North Am 36:527-542.

Sigal MJ, Aubin JE, Ten Cate AR, Pitaru S (1984). The odontoblastic process extends to the dentinoenamel junction: an immunocytochemical study of rat dentin.J Histochem Cytochem 32:872-878.
Silverstone LM (1982). The use of pit and fissure sealants in dentistry: Present status and future developments. Pediatr Dent 4:16-22.

Tanford C (1980). The hydrophobic effect: Formation of micelles and biological membranes. 2nd ed. New York: Wiley-Interscience, 23-45.

Thomas HF (1979). The extent of the odontoblast process in human dentin.J Dent Res 58:2207-2218.

Van Meerbeek B, Dhem A, Goret-Nicaise M, Braem M, Lambrechts P, Vanherle G (1993). Comparative SEM and TEM examination of the ultrastructure of the resin-dentin interdiffusion zone. J Dent Res 72:495-501.

Vanherle G, Verschueren M, Lambrechts P, Braem M (1986). Clinical investigation of dentin adhesive systems. Part I: An in vivo study.J Prosthet Dent 55:157-163.

Wang T, Nakabayashi N (1991). Effect of 2-(methacryloxy)ethyl phenyl hydrogen phosphate on adhesion todentin.JDent Res70:59-66.

Weber DF, Zaki AE (1986). Scanning and transmission electron microscopy of tubular structures presumed to be odontoblast processes. J Dent Res 65:982-986.

Yoshiyama M, Masada J, Uchida A, Ishida H (1989). Scanning electron microscope characterization of sensitive vs. insensitive human radicular dentin. J Dent Res 68:1498-1502.

Yoshiyama M, Noiri Y, Ozaki K, Uchida A, Ishikawa Y, Ishida H(1990). Transmission electron microscopic characterization of hypersensitive human radicular dentin.J Dent Res 69:1293-1297. 